\title{
A Case Series of 10 Cases of Scar Endometriosis
}

\author{
Ahmed Hassan* \\ Faculty of Medicine, Helwan University, Cairo, Egypt \\ *Correspondling author: Ahmed Hassan, Faculty of \\ Medicine, Helwan University, Cairo, Egypt
}

Received: November 21, 2018; Accepted: December 14, 2018; Published: December 21, 2018

\begin{abstract}
It is difficult to perform studies with larger series in rare condition. This is a report of my experience in managing Cesarean Scar Endometriosis (CSE) and emphasize the diagnosis and treatment options. I have collected and documented a case series of 10 patients who underwent surgical widen bloc excision for CSE. Patients' demographic features, symptoms, and clinical and operative findings were collected. The mean age was 32.4 years. Cyclical pain was documented in eight patients, while two patients presented with noncyclical pain. Menstrually-related enlargement of the nodule was observed in six patients. The mean operation time was $20 \mathrm{~min}$. The endometriotic lesions ranged from a diameter of 2 to $7 \mathrm{~cm}$ in size. Patients recovered completely, and no recurrence was observed. Four patients suffered from secondary infertility. Complete wide excision of CSE is both diagnostic and therapeutic. The most important issues to be considered during surgery is nonspreading endometriosis while manipulation. During the 12 month, follow up the four patients who suffered from infertility got pregnant.
\end{abstract}

\section{Introduction}

Endometriosis is a common gynecological condition where the endometrial glands and stromal structures are found outside the uterus. It mainly affects women in reproductive ages [1]. Endometriosis occurs most often in pelvis, on the surface lining of the pelvic cavity, peritoneum, ovaries, posterior cul-de-sac, and uterosacral ligaments. Rarely, implants of endometriosis can occur outside of the pelvis, and these forms are termed as extra pelvic endometriosis. It can also arise from scar tissues especially after cesarean section. The most accepted cause is mechanical iatrogenic implantation. Endometrial cells are inoculated directly into the surgical area and can progress to endometriosis in optimal conditions. This causes various clinical symptoms due to proliferation of these cells under the influence of female hormones. Usually there is delay in diagnosing CSE, the most common clinical symptoms and signs are swelling, tenderness on local site, and cyclic pain. Widen block excision with surrounding clear margins is both diagnostic and therapeutic intervention.

\section{Methods}

A case series of 10 patients who underwent surgical management for CSE in our obstetrics and gynecology Department - Ain Shams University. All patients were informed about surgical management and written informed consents were obtained.

All patients had a history of previous cesarean section, and their initial cesarean sections were performed in different hospitals. After the clinical assessment, the diagnosis was suspected by pelvic ultrasonography.

We performed sharp dissection with a scalpel within the area of the incision from the previous cesarean section. The cystic mass, which was surrounded by fibrosis, was removed carefully with a safe margin. The surgical intervention was followed by the reconstruction of the abdominal wall in anatomical layers.

All in cases, the definitive diagnosis was confirmed by the pathological examination.

All patients were operated under spinal anesthesia. Age, parity, body mass index, symptoms, size of tumor, time between cesarean section and the onset of symptoms, operative findings, and surgical outcomes were evaluated. Demographic features and operative findings of the cases are demonstrated in Table 1.

\section{Results}

This study includes the medical records of 10 patients who underwent surgical treatment for CSE. The first admissions of seven patients were to gynecologists, two to general surgeons, and one to a dermatologist. All were referred to our hospital. Based on medical records, Pfannenstiel incision had been performed for cesarean section in all patients.

The median age was $32.6 \pm 6.1$ years (range from 26 to 38 years), and the mean BMI was $30.95 \pm 6.59 \mathrm{~kg} / \mathrm{m}^{2}$ (range from 23.30 to $37.50 \mathrm{~kg} / \mathrm{m}^{2}$ ). The common complaint of the patients was a palpable mass under the incision scar. Eight patients suffered from cyclical pain. Noncyclic pain was seen in two patients, and four patients had experienced the enlargement of the nodule during the menstrual period. The mean time interval between initial cesarean section and the onset of symptoms was 29.2 months (range of 18 to 42 ).

The preoperative diagnosis was correct in all patients. Two patients had failed medical treatment before admission to our clinic.

All of the patients were treated surgically. Almost all the nodules were excised easily. In nine cases, extensions of the lesions through the facial layer were seen during surgery. In these cases, facial defects were repaired after excision

The mean operation time was $20.1(11-30) \mathrm{min}$. The diameter of the endometriotic lesions ranged from 2 to $7 \mathrm{~cm}$ in size. All patients were completely recovered without relapse of symptoms. The followup period was 12 months. 
Table 1:

\begin{tabular}{|c|c|c|c|c|c|c|c|}
\hline case & age & parity & BMI & Previous abdominal surgery & Asymptomatic period in months & 2ry infertility & Operative time in $\min$ \\
\hline 1 & 32 & 1 & 25 & CS & 24 & no & 25 \\
\hline 2 & 28 & 2 & 30 & $\operatorname{cs}(2)$ & 18 & no & 15 \\
\hline 3 & 30 & 1 & 33 & CS & 40 & yes & 20 \\
\hline 4 & 36 & 1 & 29 & $\mathrm{CS}$ & 42 & yes & 11 \\
\hline 5 & 26 & 1 & 31 & CS & 18 & no & 25 \\
\hline 6 & 34 & 3 & 34 & $\operatorname{cs}(3)$ & 24 & no & 30 \\
\hline 7 & 31 & 2 & 37 & $\operatorname{CS}(2)$ & 24 & no & 15 \\
\hline 8 & 32 & 1 & 23 & CS, appendictomy & 30 & yes & 20 \\
\hline 9 & 38 & 2 & 35 & CS (2) & 48 & no & 15 \\
\hline 10 & 33 & 1 & 24 & $\mathrm{CS}$ & 24 & yes & 25 \\
\hline
\end{tabular}

Asymptomatic period was defined as the time interval between the previous surgery and the onset of the symptoms. Number in the parentheses indicates the number of procedures.

No recurrence was observed during the follow-up period. Four patients suffered from 2ry infertility and they got pregnant spontaneously during the first year after the operation.

\section{Discussion}

Endometriosis in cesarean scar is a rarely observed finding. There are a limited number of publications discussing CSE, and most of them are case reports. It is difficult to conduct well-controlled clinical trials in rarely observed disease. The common symptom of CSE includes palpable mass, typically accompanied by cyclic, noncyclic, or constant pain. Menstruation usually aggravates disease. The history and complain of the patient helps in diagnosis of the illness. De Oliveira et al. reported a case-control study to identify the risk factors of scar endometriosis. According to this study, early hysterotomy in pregnancy especially before $22^{\text {nd }}$ week of gestation is the main risk factor [2]. Additionally, increased menstrual flow and alcohol consumption are also concluded as risk factors, while high parity may be a protecting factor [2]. The most common risk factor for the presence of endometriosis in scar tissue is a previous history of obstetric surgical procedures [3]. The reason was defined by Wang et al. first of all, obstetric surgery can expose a large amount of endometrial cells, and these cells can be entrapped in the wound [4]. The separation of active cells may be facilitated by amniotic fluid and significantly, more blood loss in obstetric surgery would provide a relatively rich nutritional environment for the growth of endometrial tissue in the wound [4]. In this study, more than half of our patients were overweight. This can provide wide surgical surface for the entrapment of endometrial active cells and may be an impact on the illness. In this study, the time interval between cesarean section and the onset of symptoms is ranged from 18 months to 42 months. The relative late onset of symptoms after surgery is the probable cause of misdiagnosis [1]. The mean duration of the symptoms was higher in patients whom first admission was not to the obstetrician. Overall, general surgeons are infrequently involved in the management of cesarean section scar lesions [5].

History and physical examination is essential for an accurate diagnosis. Scar endometriosis is usually developed in superficial layers of the connective tissue, and nodules are usually found by palpation. The clinical evaluation can be confirmed by pelvic ultrasonography. Ultrasound is the most accessible, reliable, and cost-effective procedure. Some additional diagnostic procedures such as fine-needle aspiration, computed tomography, and magnetic resonance imaging can be performed $[5,6]$. The imaging modalities are nonspecific and more useful for differential diagnoses and detecting the relationship between the mass and the other tissues. These are also used in planning of operative resection, to identify and to evaluate the extent of disease. Awareness of its typical clinical manifestations remains the mainstay for intervention and diagnosis.

In this study, all cesarean sections were performed outside of our hospital. Hence, we could not contribute to the literature about the incidence of CSE. It can be assumed as a methodological limitation of our study. Based on literature reviewed, the estimated incidence of scar endometrioma ranges from 0.02 to $3.4 \%$ and more frequently observed with an incidence of 0.03 to $0.47 \%$ following cesarean delivery [3,7].

Nominato et al. suggested that cesarean section is the commonest cause of developing scar endometriosis [8]. It should be suspected in any patient, with scar-related masses, who had a history of uterine surgery, especially cesarean section.

However, endometriosis on the abdominal wall can be observed in some sporadic cases that have not been previously exposed to any type of surgery [9]. Furthermore, some presentations of abdominal wall endometriomas are referred to surgery performed by general surgeons such as appendectomy [10]. In these cases, hematogenous outspread, lymphatic dissemination, coelomic metaplasia, and some else theories could be addressed for probable pathogenesis. In our study, all cases had a history of at least one cesarean section. By the fact that the number of cesarean sections is constantly increasing, this complication becomes more frequent [11-13].

In this case, series, we did not find any evidence of pelvic endometriosis. Iatrogenic mechanical transplantations on incision scars during the operations are the most accepted pathogenesis. So it is important to take some precautions to avoid transplantation of endometrial cells. To minimize endometriosis contamination, some authors recommend careful isolation of the wall incision and lavage with saline before the closure of the wall [14]. The others hypothesized that failure to close the parietal and visceral peritoneum with sutures at the time of cesarean section may markedly increase 
the postoperative occurrence of an endometrioma in the skin incision scar [15].

Replacing instruments and needles with a new one is recommended when suturing other abdominal layers [16]. Based on literature reviewed, we did not find any correlation between the indications of cesarean section and development of CSE. On the other hand, some authors reported the correlations with timing of cesarean section and CSE.

Wicherek et al. stated that cesarean section performed before spontaneous onset of labor may increase substantially the risk of occurrence of scar endometriomas [17]. Immune tolerance during pregnancy was suggested to be an important factor predisposing to the implantation [17]. Similarly in this study, 7 of 10 patients had cesarean section without the presence of regular uterine contractions. In other words, seven cases were elective. Medical treatment gives only partial relief of pain and with regard to the almost certain recurrence of the condition after cessation of medication $[5,18]$. The use of progestogens, oral contraceptive pills, and danazol are not effective [5].

Due to side effects of androgens, patients have poor compliance to these drugs [5]. Two of our patients had a history of medical treatment failure before admission to the hospital. Treatment of choice is wide excision. Surgical treatment is overly recommended $[1,19,20]$.

The mean operation time was $20 \mathrm{~min}$. None of them have required further surgical intervention in the follow-up. So in order to prevent the recurrences, surgical excision remains the preferable method. Local recurrence is likely to be after an inadequate surgical excision $[1,20]$.

Four patients in this study achieved spontaneous pregnancy within the first year after the excision of the CSE, which suggest that scar endometriosis may have biochemical effect that affects the process of ovulation and implantation, further studies needed to confirm this finding as no data in the literature to support this observational finding.

\section{References}

1. Gupta P, Gupta S. Scar Endometriosis: a Case Report with Literature Review. Acta Med Iran. 2015; 53: 793-795.

2. Vellido-Cotelo R, Muñoz-González JL, Oliver-Pérez MR, de la Hera-Lázaro C, Almansa-González C, Pérez-Sagaseta C, et al. Endometriosis node in gynaecologic scars: a study of 17 patients and the diagnostic considerations in clinical experience in tertiary care center. BMC Womens Health. 2015; 15 : 13-15
3. Vural B, Vural F, Müezzinoglu B. An Abdominal Wall Desmoid Tumour Mimicking Cesarean Scar Endometriomas: A Case Report and Review of the Literature. J Clin Diagn Res. 2015; 9: 14-15.

4. Uçar MG, Şanlıkan F, Göçmen A. Surgical Treatment of Scar Endometriosis Following Cesarean Section, a Series of 12 Cases. Indian J Surg. 2015; 77 : 682-686.

5. Padmanabhan LD, Mhaskar R, Mhaskar A. Scar endometriosis. J Obstet Gynaecol India. 2003; 53: 59-60.

6. Bhowmick RN, Paul P, Dutta S. Endometriosis of laparotomy scar. J Obstet Gynaecol India. 1986; 36: 130-131.

7. Chatterjee SK. Scar endometriosis: A Clinicopathological study of 17 cases. Obstet Gynecol. 1980; 56: 81-84.

8. Nirula R, Greaney GC. Incisional endometriosis: An under appreciated diagnosis in general surgery. Am Coll Surg. 2000; 190: 404-407.

9. Ideyi SC, Schein M, Niazi M. Spontaneous endometriosis of the abdominal wall. Dig Surg. 2003; 20: 246-248.

10. Tomas E, Martin A, Garfia C, Gomez FS, Morillas JD, Tortajada GC. Abdominal wall endometriosis in absence of previoussurgery. J Ultrasound Med. 1999; 18: 373-375.

11. Demiral G, Aksoy F, Ozclik A, Saban B, Kusak M, Ekinci O, Erengul C Cesarean scar endometriosis: presentation of eleven clinical cases and review of the literature. J Turk Soc Obstet Gynecol. 2011; 8: 209- 213.

12. Elabsi M, Lahlou MK, Rouas L, Essadel H, Benamer S, Mohammadi A, et al. Cicatrix endometriosis of the abdominal wall. Ann Chir. 2002; 127: 65-67.

13. Phupong V, Triratanachat S. Ceserean section scar endometriosis: a case report and review of the literature. J Med Assoc Thai. 2002; 85: 733-734.

14. Zhang J, Liu X. Clinicopathological features of endometriosis in abdominal wall-clinical analysis of 151 cases. Clin Exp Obstet Gynecol. 2016; 43: 379383.

15. Akdemir A, Akman L, Yavuzsen HT, Zekioglu O. Clinical features of patients with endometriosis on the cesarean scar. Kaohsiung J Med Sci. 2014; 30: 541-543.

16. Blanco RG, Parithivel VS, Shah AK, Gumbs MA, Schein M, Gerst PH Abdominal wall endometriomas. Am J Surg. 2003; 185: 596-597.

17. Ozler A, Yaldız S, Değirmencioglu Al. Karın duvarı endometriozisi: Olgu sunumu. Dicle Tip Derg. 2010; 37: 410.

18. Andolf $\mathrm{E}$, Thorsell M, Kallen K. Caesarean section and risk for endometriosis: a prospective cohort study of Swedish registries. BJOG. 2013; 120: 10611065.

19. Medeiros F, Cavalcante DI, Medeiros MA, Eleutério J. Fine-needle aspiration cytology of scarendometriosis: study of seven cases and literature review. Diagn Cytopathol. 2011; 39: 18-21.

20. Veda P, Srinivasaiah M. Incisional endometriosis: diagnosed by fine needle aspiration cytology. J Lab Phys. 2010: 2; 117-120.
Austin J Obstet Gynecol - Volume 5 Issue 9 - 2018

Submit your Manuscript | www.austinpublishing group.com

Hassan. (C) All rights are reserved
Citation: Hassan A. A Case Series of 10 Cases of Scar Endometriosis. Austin J Obstet Gynecol. 2018; 5(9): 1130. 